\title{
Myeloid SIRT1 regulates macrophage infiltration and insulin sensitivity in mice fed a high-fat diet
}

\author{
Sun-O Ka, Mi-Young Song, Eun Ju Bae ${ }^{1}$ and Byung-Hyun Park
}

Department of Biochemistry, Chonbuk National University Medical School, 567 Baekje-daero, Deokjin-gu, Jeonju, Jeonbuk 561-756, Republic of Korea

${ }^{1}$ College of Pharmacy, Woosuk University, 443 Samnye-ro, Wanju, Jeonbuk 565-701, Republic of Korea

Correspondence should be addressed to B-H Park or E J Bae Emails bhpark@jbnu.ac.kr or ejbae@woosuk.ac.kr

\begin{abstract}
Inflammation is an important factor in the development of insulin resistance. SIRT1, a class 3 histone/protein deacetylase, has anti-inflammatory functions. Myeloid-specific deletion of Sirt1 promotes macrophage infiltration into insulin-sensitive organs and aggravates tissue inflammation. In this study, we investigated how SIRT1 in macrophages alters tissue inflammation in the pancreas as well as liver and adipose tissue, and further explored the role of SIRT1 in locomotion of macrophages. Myeloid-specific Sirt1-deleted mice (mS1KO) and WT littermates were fed a 60\% calorie high-fat diet (HFD) for 16 weeks. Tissue inflammation and metabolic phenotypes were compared. Bone marrow macrophages (BMMs) from WT or mS1KO mice were used in in vitro chemotaxis assays and macrophage polarization studies. mS1KO mice fed a HFD exhibited glucose intolerance, reduced insulin secretion, and insulin sensitivity with a slight decrease in body weight. Consistent with these results, pancreatic islets of mS1KO mice fed a HFD displayed decreased mass with profound apoptotic cell damage and increased macrophage infiltration and inflammation. Liver and adipose tissues from mS1KO HFD mice also showed greater accumulation of macrophages and tissue inflammation. Results from in vitro experiments indicated that deletion of myeloid Sirt1 stimulated proinflammatory M1-like polarization of BMMs and augmented the adipocyte-mediated macrophage chemotaxis. The latter effect was accompanied by increased expression and acetylation of focal adhesion kinase, as well as nuclear factor kappa B.

Our results indicate that myeloid SIRT1 plays a crucial role in macrophage polarization and chemotaxis, and thus regulates the development of HFD-induced pancreatic inflammation and insulin secretion, and metabolic derangements in liver and adipose tissue.
\end{abstract}

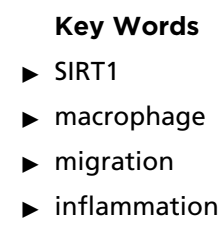

Journal of Endocrinology (2015) 224, 109-118

\section{Introduction}

Obesity is characterized by chronic low-grade tissue inflammation that contributes to the development of insulin resistance, metabolic syndrome, and type 2 diabetes (Hotamisligil 2006). Infiltration of immune cells into peripheral tissues and the consequent tissue inflammation are responsible for obesity-related insulin resistance. Macrophages are infiltrating immune cells that are central to initiating and orchestrating obesity-induced local inflammation. The importance of macrophages in inflammatory responses and subsequent metabolic derangements has been well documented in genetic studies. Transgenic overexpression of monocyte chemotactic 
protein 1 (C-C chemokine ligand 2 (CCL2)) in adipocytes increases macrophage infiltration and decreases insulin sensitivity (Kanda et al. 2006). In contrast, genetic deletion of C-C chemokine ligand $2(\mathrm{Ccl} 2) / \mathrm{Mcp} 1$ or its receptor, C-C chemokine receptor 2 ( $\mathrm{Ccr} 2)$, protects mice from high-fat-diet (HFD)-induced inflammation and insulin resistance (Kanda et al. 2006, Weisberg etal. 2006). Similarly, macrophage-specific deletion of I $\mathrm{B}$ kinase- $\beta$ (Ikbkb) or c-Jun N-terminal kinase (Mapk8) suppresses inflammatory pathways and improves systemic insulin sensitivity in mice on a HFD (Arkan et al. 2005, Solinas et al. 2007).

SIRT1, an $\mathrm{NAD}^{+}$-dependent histone deacetylase, is an important regulator of the metabolic response to caloric restriction (Chalkiadaki \& Guarente 2012). Additional evidence indicates that SIRT1 represses inflammatory signaling in multiple tissues and cell types, including macrophages. Caloric restriction increases the levels of SIRT1 protein in peritoneal macrophages and suppresses the production of proinflammatory mediators (Clement et al. 2004, Zhang et al. 2010). Similarly, siRNAmediated Sirt1 knockdown in RAW264.7 cells increases tumor necrosis factor alpha (TNF $\alpha$ ) secretion (Shen et al. 2009), and genetic or pharmacological activation of SIRT1 suppresses cytokine release from stimulated macrophages (Yoshizaki et al. 2010, Zhang et al. 2010). Myeloid-cellspecific deletion of Sirt1 increases macrophage infiltration into the liver and adipose tissues, as well as production of proinflammatory cytokines, and exacerbates insulin resistance after high-fat feeding (Schug et al. 2010). Taken together, these findings indicate a close link between the activity of SIRT1 in macrophages and obesity-induced inflammation. However, the mechanism through which SIRT1 regulates macrophage locomotion in response to metabolic stresses remains unclear.

Cell migration is a complicated process regulated by the activation of various signaling molecules. Results from several studies have indicated that nuclear factor kappa $\mathrm{B}$ (NFKB (NFKB1)) is a central coordinator of macrophage migration in obesity-induced inflammation models both in vitro and in vivo (Suganami et al. 2007, Ichioka et al. 2011, Le et al. 2011). Notably, SIRT1 deacetylates the p65 subunit of NFKB1 and attenuates NFKB1-mediated gene transcription (Lee et al. 2009). Specifically, myeloid-cellspecific deletion of Sirt1 results in hyperactivation of the NFKB1 pathway, and, in mice, leads to the development of systemic insulin resistance (Schug et al. 2010), indicating that SIRT1 might regulate macrophage migration by targeting NFKB1.

The intracellular non-receptor tyrosine kinase, focal adhesion kinase (FAK), modulates cell adhesion and migration through integrin signaling (Parsons 2003). Fak (Ptk2) transcription is regulated by binding of NFKB1 to the Ptk2 gene promoter (Golubovskaya et al. 2004). Therefore, the hypothesis has been proposed that SIRT1 might regulate macrophage migration by targeting the NFKB1 and FAK pathways. To test this hypothesis, we examined the migratory ability of Sirt1-deleted macrophages in vitro, as well as macrophage infiltration in myeloid-cell-specific Sirt1 knockout (mS1KO) mice under HFD conditions.

\section{Materials and methods}

\section{Animal experiments}

Sirt $1^{\text {flox/flox }}$ mice were crossed with LysM-Cre mice to generate mS1KO mice. SIRT1 KO mice and age-matched littermates older than 4 weeks were provided with either a standard laboratory chow diet or a $60 \%$ HFD and allowed to feed ad libitum (Research Diet, New Brunswick, NJ, USA) for 16 weeks. Oral glucose tolerance tests ( $1 \mathrm{~g} / \mathrm{kg}$ of body weight) and insulin tolerance tests ( $0.75 \mathrm{U} / \mathrm{kg}$ of body weight) were performed after $14 \mathrm{~h}$ of fasting. All experimental procedures were approved by the Institutional Animal Care and Use Committee of Chonbuk National University.

\section{Histology}

Fixed tissues were embedded in paraffin. Tissue sections ( 4 or $6 \mu \mathrm{m}$ ) were stained with hematoxylin and eosin (H\&E) or Sirius red for light microscopy. For immunohistochemistry, sections were immunostained with antibodies against insulin (Santa Cruz Biochemicals, Santa Cruz, CA, USA) or F4/80 (Abcam, Cambridge, UK). The adipocyte area and islet size in sections were measured using the iSolution DT 36 Software (Carl Zeiss, Oberkochen, Germany). Liver inflammation in liver biopsies was graded using a modified histologic activity index (Kleiner et al. 2005). TUNEL staining was carried out using a commercial kit (Promega).

\section{Cell culture}

Bone marrow-derived macrophages (BMMs) were generated using mouse macrophage colony-stimulating factor. For M1 or M2 differentiation, BMMs were treated with LPS $(10 \mathrm{ng} / \mathrm{ml})+\mathrm{IFN} \gamma(50 \mathrm{U} / \mathrm{ml}$, Invitrogen $)$ or IL4 $(10 \mathrm{ng} / \mathrm{ml}$, Invitrogen) respectively. RAW264.7 macrophage cells were treated with SIRT1 activator (10 nM SRT1720) or inhibitor $(2.5 \mu \mathrm{M}$ sirtinol $)$ for $24 \mathrm{~h}$. To prepare 3T3-L1 conditioned medium $(\mathrm{CM}), 3 \mathrm{~T} 3-\mathrm{L} 1$ cells were cultured

Published by Bioscientifica Ltd 
for 2 days after completion of differentiation. To express exogenous proteins for immunoprecipitation, 293T cells were transfected with pFlag-Sirt1 or p300 using Lipofectamine 2000 (Invitrogen).

\section{In vitro migration assay}

BMM or RAW264.7 cell migration assays were carried out in transwell migration assay chambers (BD Life Sciences, Franklin Lakes, NJ, USA) by adding CCL2 or CM to the lower chamber.

\section{Statistical analysis}

Data are expressed as mean \pm s.E.M. Statistical comparisons were made using one-way ANOVA followed by Fisher's post hoc analysis. The significance of differences between groups was determined using Student's unpaired $t$-test. A $P$ value of $<0.05$ was considered significant.

\section{Additional methods}

Detailed methods are provided in the Supplementary Methods, see section on supplementary data given at the end of this article.

\section{Results}

\section{Myeloid Sirt1 deletion leads to impaired glucose tolerance after high-fat feeding}

To evaluate the functional role of myeloid SIRT1 in the development of obesity-associated tissue inflammation, we generated $\mathrm{mS} 1 \mathrm{KO}$ mice in a C57BL/6 background (Supplementary Fig. 1a, see section on supplementary data given at the end of this article and Supplementary Table 1). Western blotting confirmed complete deletion of Sirt1 in BMMs from mS1KO mice (Supplementary Fig. 1b). Four-week-old mS1KO mice and WT littermates were fed either a normal chow diet (NCD) or 60\% HFD for 16 weeks. On a NCD, there were no differences in body weight gain, food intake, body fat mass, plasma TG, or cholesterol level between WT and mS1KO mice (Supplementary Fig. 2a, b, c and d). However, on a HFD, mS1KO mice showed decreased body weight at 10 and 16 weeks after HFD feeding and had lower levels of plasma cholesterol, compared with WT mice (Supplementary Fig. 2a and d).

In addition, all parameters, such as fasting blood glucose, insulin levels, glucose tolerance, and insulin tolerance results were the same for WT and $\mathrm{mS} 1 \mathrm{KO}$ mice
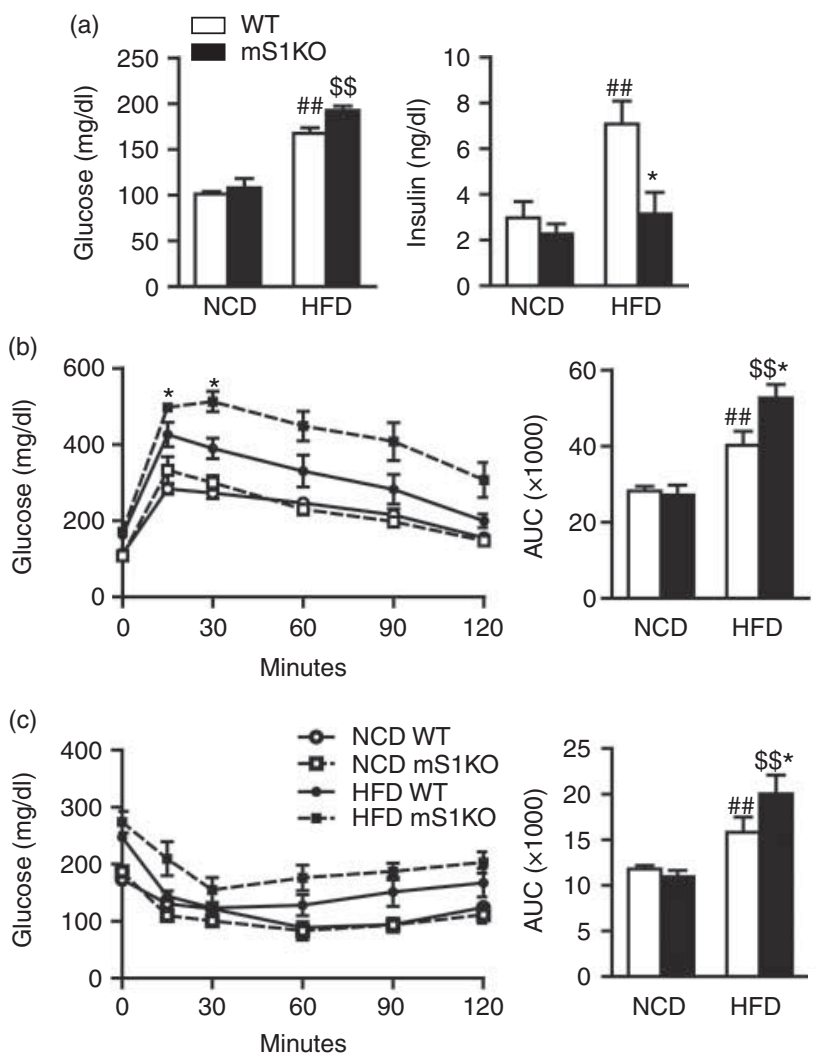

\section{Figure 1}

Metabolic characteristics of mS1KO mice fed a HFD. WT or mS1KO mice were fed with either a NCD or a HFD for 16 weeks. (a) Fasting plasma glucose and insulin levels. Plasma glucose concentrations during i.p. glucose tolerance tests (b) and insulin tolerance tests (c) in overnight fasted mice. Areas under the curve were compared. Values are means \pm S.E.M. $(n=6) .{ }^{\# \#} P<0.01$ versus WT NCD; ${ }^{\$} P<0.01$ versus $\mathrm{mS1KO} N C D$; and ${ }^{*} P<0.05$ versus WT HFD.

on a NCD (Fig. 1). In contrast, on a HFD mS1KO mice exhibited higher fasting glucose levels, with markedly reduced plasma insulin levels and impaired glucose tolerance, indicating that development of glucose intolerance is secondary to defective insulin secretion in mS1KO mice (Fig. 1a and b). Results of insulin-tolerance tests indicated that $\mathrm{mS} 1 \mathrm{KO}$ mice fed a HFD have reduced insulin sensitivity compared with WT mice (Fig. 1c).

\section{mS1KO mice display pancreatic dysfunction with islet atrophy and inflammation}

To understand why mS1KO mice exhibited glucose intolerance despite having body weights similar to WT mice, and to determine whether the reduction in the levels of insulin in plasma was due to primary dysfunction of the pancreas, we focused on pancreatic islets. Histological examination of pancreatic tissue by H\&E staining and insulin immunostaining revealed that deletion of myeloid

Published by Bioscientifica Ltd. 
(a)

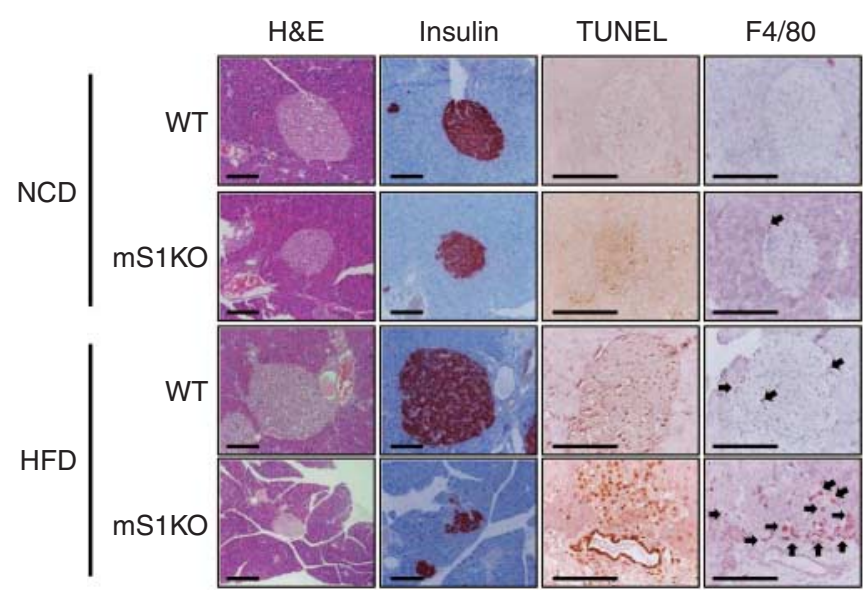

(b)

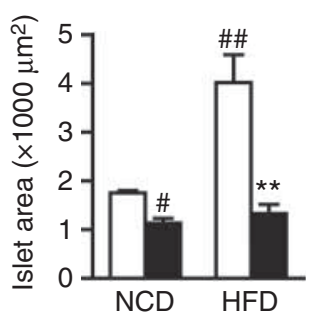

(d)

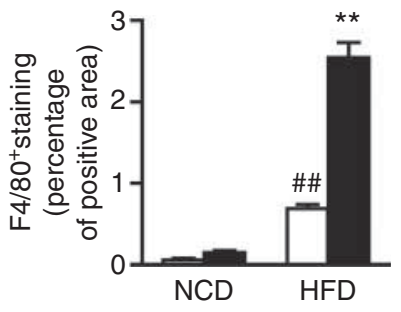

(c)

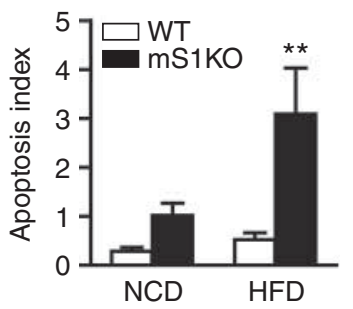

(e)

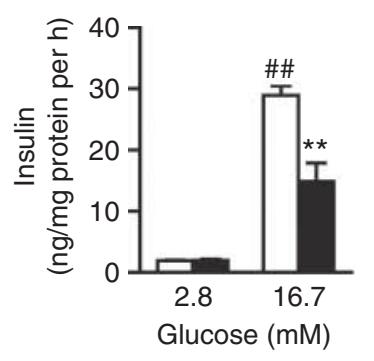

Figure 2

Effects of deletion of myeloid Sirt1 on pancreatic infiltration of macrophages. (a) Pancreases were retrieved 16 weeks after HFD feeding and stained with H\&E or immunostained with antibodies against insulin or $\mathrm{F} 4 / 80$. Bars $=250 \mu \mathrm{M}$. For apoptosis analysis, TUNEL staining was performed. Arrows indicate infiltrated macrophages. (b, c and d) Islet area,

Sirt1 resulted in a decrease in islet mass under NCD conditions. HFD feeding induced pancreatic islet hyperplasia in WT mice, but the islet mass reduction was even more aggravated in mS1KO mice (Fig. 2a and b). Consistent with these results, the apoptotic index, analyzed by TUNEL assay, was higher in $\mathrm{mS1KO}$ mice receiving a HFD (Fig. 2a and c). To assess macrophage infiltration into islets, we counted F4/80-positive cells as a pan-marker for macrophages. Accumulation of cells immunopositive for F4/80 was significantly increased in mS1KO mice fed a HFD compared with WT mice; mS1KO mice also displayed increased intra-islet and peri-islet inflammatory cell infiltration after HFD feeding (Fig. 2a and d). We measured glucose-stimulated insulin secretion by islets isolated from WT and mS1KO mice. Islets from mS1KO mice exhibited a marked repression in high-glucose-stimulated insulin secretion, consistent with the in vivo results in Fig. 1A (Fig. 2e). Basal insulin release under low-glucose conditions was similar between the genotypes.

\section{Deletion of myeloid Sirt1 increases macrophage infiltration into liver and adipose tissue}

Increased macrophage infiltration in peripheral tissues such as liver and adipose tissue is a hallmark of obesityinduced tissue inflammation and insulin resistance. apoptosis, and $\mathrm{F} 4 / 80^{+}$macrophage infiltration were analyzed. (e) Islets were isolated from 12-week-old mice, and glucose-stimulated insulin secretion was quantified by ELISA. Insulin level was normalized to total protein content. Values are means \pm s. E.M. $(n=6)$. ${ }^{\#} P<0.05$, and ${ }^{\# \#} P<0.01$ versus WT NCD and ** $P<0.01$ versus WT HFD.

Given that increased numbers of macrophages infiltrated into the pancreas in $\mathrm{mS} 1 \mathrm{KO}$ mice on a HFD, we then examined macrophage infiltration into the liver and adipose tissue, and the mRNA expression of inflammatory genes. Liver weight and TG content were similar between genotypes in mice on a NCD, but significantly decreased in mS1KO mice on a HFD compared with WT mice on the same diet (Fig. 3a). Examination of liver histology by microscopy with $\mathrm{H} \& \mathrm{E}$ staining revealed a higher grade of inflammation in $\mathrm{mS} 1 \mathrm{KO}$ mice than WT mice under both NCD and HFD conditions (Fig. 3b). Liver tissue damage in $\mathrm{mS} 1 \mathrm{KO}$ mice, as evidenced by increases in serum ALT and AST levels, correlated well with the degree of inflammation (Fig. 3c). Real-time RT-PCR analysis also confirmed the increased accumulation of macrophages and inflammation in mS1KO mice compared with WT mice (Fig. 3d).

Feeding with a HFD induced a large increase in the mass of epididymal white adipose tissue (eWAT) in WT mice but had less effect in $\mathrm{mS} 1 \mathrm{KO}$ mice, resulting in lower eWAT in mS1KO mice on a HFD than in WT HFD mice (Fig. 4a). In agreement with this finding, adipocyte size was also smaller in $\mathrm{mS} 1 \mathrm{KO}$ mice (Fig. $4 \mathrm{~b}$ and $\mathrm{c}$ and Supplementary Fig. 3a, see section on supplementary data given at the end of this article). Adipose tissue macrophages (ATMs) often surround and ingest dying

Published by Bioscientifica Ltd. 
(a)
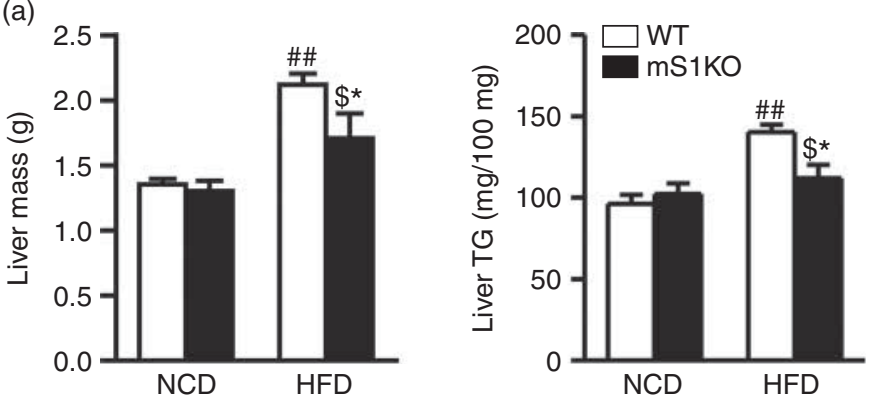

(b)
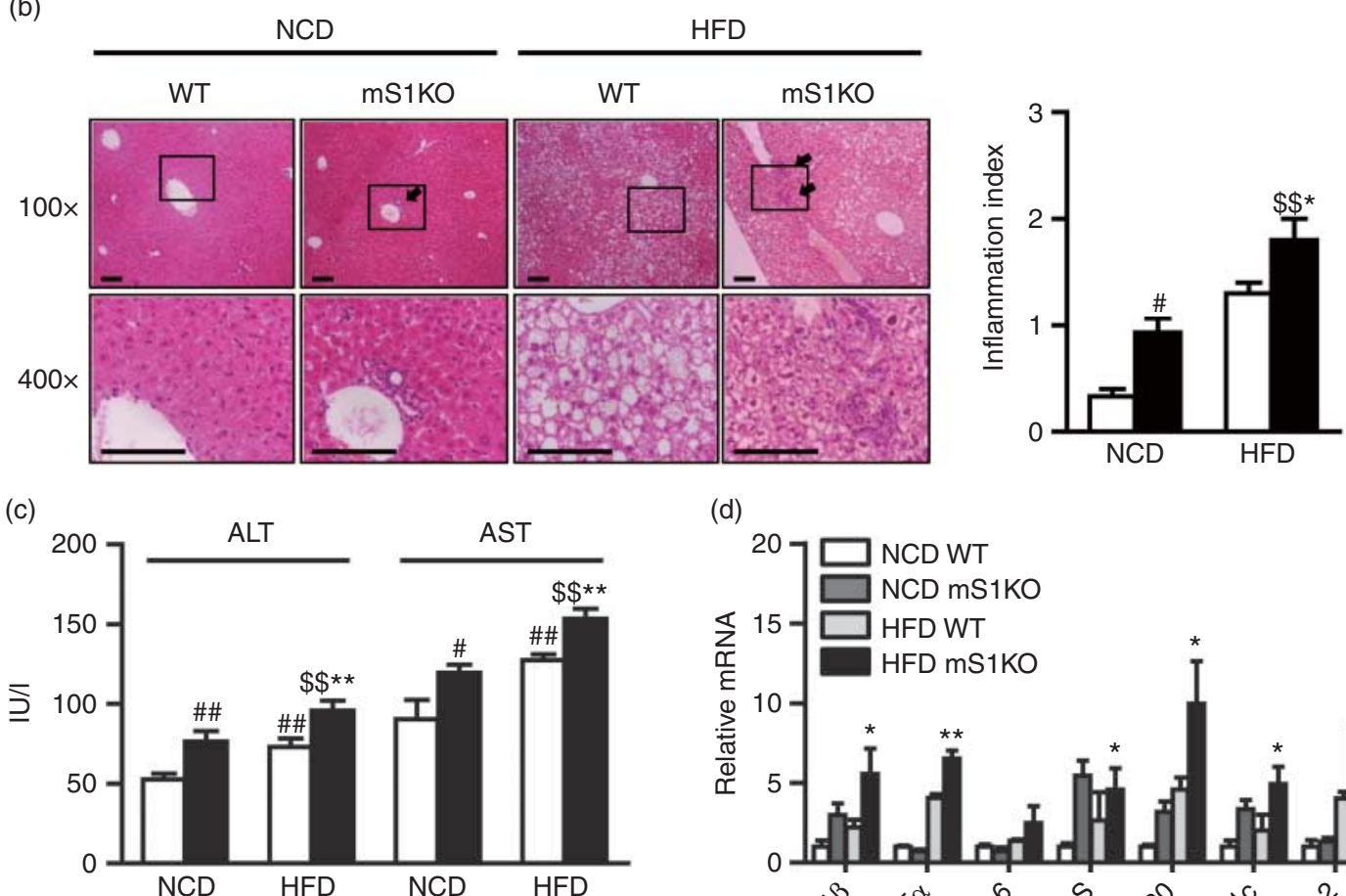

(d)

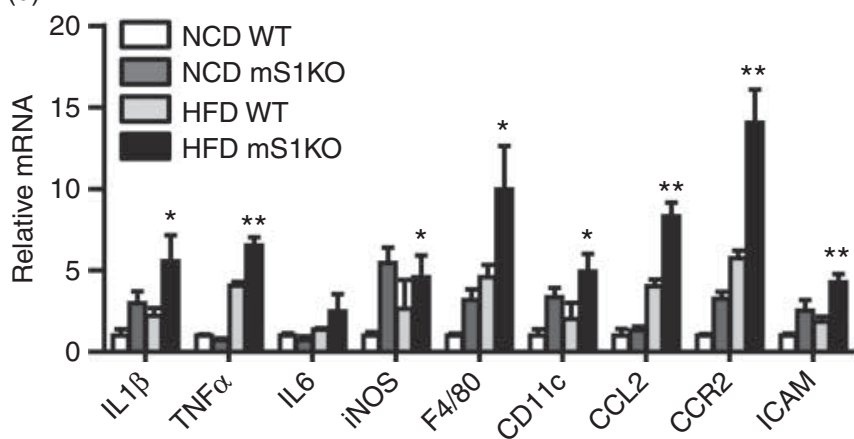

Figure 3

Effects of deletion of myeloid Sirt1 on hepatic infiltration of macrophages. (a) Liver mass and liver TG were determined. (b) Liver tissues were retrieved 16 weeks after HFD feeding and subjected to H\&E staining. Arrows indicate inflammatory cell clusters. Inflammation scores were determined. Bars $=250 \mu \mathrm{m}$. (c) Plasma levels of ALT and AST were measured by

specific ELISA. (d) Expression of macrophage-infiltration-related genes was determined by real-time RT-PCR. Values are means \pm S.E.M. $(n=6) .{ }^{\#} P<0.05$, ${ }^{\# \#} P<0.01$ versus WT NCD; ${ }^{\$} P<0.05$, and ${ }^{\$ \$} P<0.01$ versus $\mathrm{mS} 1 \mathrm{KO} N C D$; and $* P<0.05$, and $* * P<0.01$ versus WT HFD.

or dead adipocytes to form crown-like structures (CLSs). To assess macrophage infiltration into eWAT, we counted the numbers of CLSs in the tissue. As shown in Fig. $4 \mathrm{~b}$ and $\mathrm{d}, \mathrm{mS} 1 \mathrm{KO}$ mice had more CLSs, suggesting that ATM content was increased in mS1KO mice even though they have less adipose tissue. To characterize the ATMs, we prepared stromal vascular fractions from adipose tissues of HFD WT and $\mathrm{mS} 1 \mathrm{KO}$ mice and analyzed them by FACS. FACS analysis revealed that HFD feeding resulted in a higher percentage of $\mathrm{F} 4 / 80^{+} \mathrm{CD} 11 \mathrm{~b}^{+} \mathrm{CD} 11 \mathrm{c}^{+}$ macrophages in adipose tissue of $\mathrm{mS} 1 \mathrm{KO}$ mice than in
WT mice (Fig. 4e and Supplementary Fig. 3b). Consistent with this finding, levels of mRNAs of a variety of proinflammatory genes, including cytokines/chemokines, were upregulated, while the level of a representative anti-inflammatory adipokine, adiponectin, was downregulated, in mS1KO HFD mice (Supplementary Fig. 3c). The secretion of cytokines, including the chemokine CCL2, was also significantly increased in mS1KO HFD mice (Fig. 4f). Similar to the findings observed for pancreatic islets, more TUNEL-positive apoptotic cells were observed in the adipose tissue of $\mathrm{mS} 1 \mathrm{KO}$ mice 

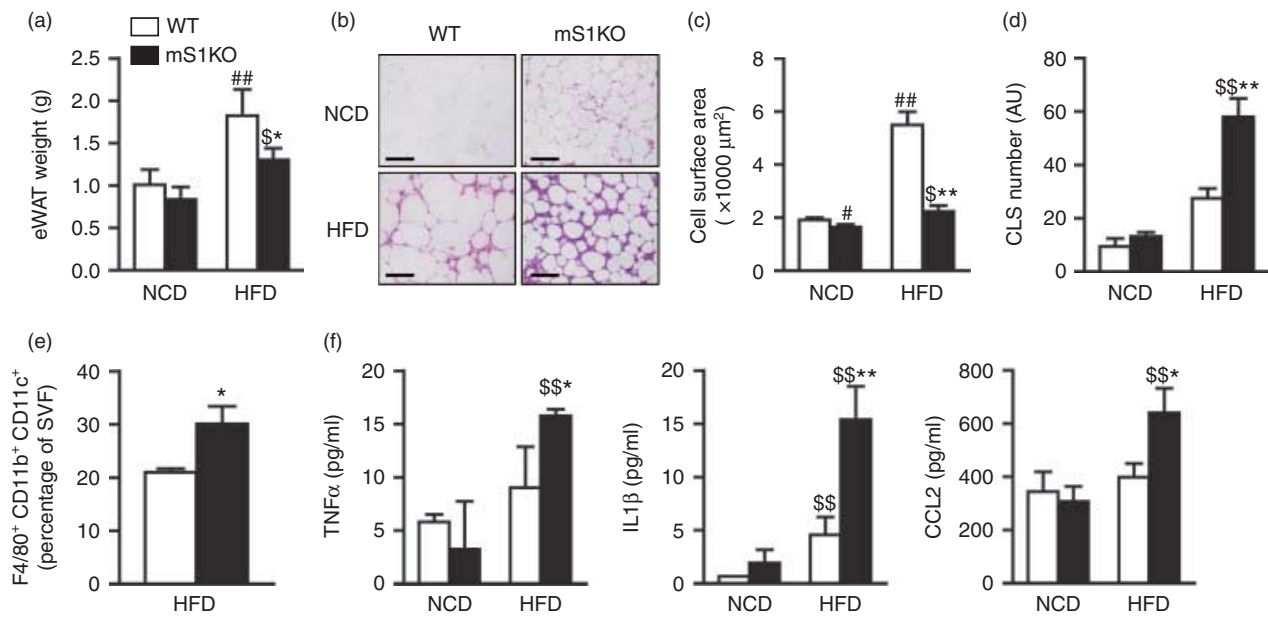

\section{Figure 4}

Effects of deletion of myeloid Sirt1 on adipose tissue infiltration by macrophages. (a) Weight of eWAT from WT and mS1KO mice fed a NCD or a HFD for 16 weeks. (b, c and d) eWAT was stained with H\&E, and the mean surface area of adipocytes and the numbers of crown-like structures (CLSs) were determined. Bars $=250 \mu \mathrm{m}$. (e) The macrophage subpopulation was

(Supplementary Fig. 3d). It is well known that infiltrated macrophages produce transforming growth factor $\beta$ and induce tissue fibrosis in adipose tissue (Olefsky \& Glass 2010). To further examine the characteristics of adipose tissues, tissue sections were stained with Sirius red and the representative sections were quantified by digital image analysis. There was an increase in Sirius red staining in the stroma of adipose tissues in WT HFD mice compared with those on a NCD. Sirius red staining around individual adipocytes was markedly increased in mS1KO HFD mice, which indicates a large increase in collagen fiber content of adipose tissues (Supplementary Fig. 3d).

\section{Deletion of myeloid Sirt1 increases macrophage migration}

In response to specific environmental stimuli, ATMs polarize to classically activated proinflammatory M1-like cells or alternatively activated, less inflammatory M2-like cells (Lumeng et al. 2007). We examined changes in the levels of mRNA for SIRT1 in M1 and M2 macrophages. Expression of SIRT1 was suppressed in M1 macrophages and increased in M2 macrophages (Fig. 5a), supporting the anti-inflammatory role of SIRT1. Furthermore, the expressions of CCL2 and proinflammatory genes such as Tnf $\alpha$ (Tnf), Il6, and iNos (Nos2) were significantly increased in M1 macrophages of $\mathrm{mS} 1 \mathrm{KO}$ mice compared with those of WT mice, whereas M2 marker genes were similarly expressed in M2 macrophages from both genotypes (Fig. 5b). analyzed by FACS. (f) Plasma levels of TNF $\alpha$, IL1 $\beta$, and CCL2 were determined by ELISA. Values are means \pm s.E.M. $(n=6)$. ${ }^{\#} P<0.05$, and ${ }^{\# \#} P<0.01$ versus WT NCD; ${ }^{\$} P<0.05$, and ${ }^{\$ \$} P<0.01$ versus $\mathrm{mS1KO}$ NCD; and $* P<0.05$, and $* * P<0.01$ versus WT HFD.

To examine the underlying mechanisms of the enhanced infiltration of macrophages and inflammation in tissues of $\mathrm{mS} 1 \mathrm{KO}$ mice, we directly assessed the genetic or chemical effects of inhibition or activation of SIRT1 on macrophage migration using an in vitro chemotaxis assay. When adipocyte CM or CCL2 was used as a chemoattractant, BMMs from $\mathrm{mS} 1 \mathrm{KO}$ mice showed increased migration compared with those from WT mice (Fig. 5c). Consistent with this result, treatment of RAW264.7 cells with SRT1720, a SIRT1 activator, suppressed CM-mediated macrophage migration. inhibition of SIRT1 with sirtinol enhanced the effect of $\mathrm{CM}$ on macrophage migration (Fig. 5d).

\section{Upregulation of NFKB1/FAK pathways and involvement in macrophage migration in $\mathrm{mS} 1 \mathrm{KO}$ mice}

Activation of NFKB1 and FAK has been implicated in cell migration (Maa et al. 2008) and FAK expression is regulated by NFKB1 (Golubovskaya et al. 2004). Therefore, we then investigated the involvement of NFKB1 and FAK activation in LPS-stimulated BMMs. LPS treatment induced a transient increase in acetylation of the p65 NFKB1 subunit in WT BMMs, but this change was more prominent in $\mathrm{mS} 1 \mathrm{KO}$ cells (Fig. 6a). LPS-stimulated nuclear translocation of the p65 subunit was also increased in $\mathrm{mS1KO}$ cells relative to that in WT cells. Moreover, FAK expression was clearly increased in $\mathrm{mS} 1 \mathrm{KO}$ cells at the levels of both protein and mRNA (Fig. 6b). To further investigate the role of SIRT1

Published by Bioscientifica Ltd. 

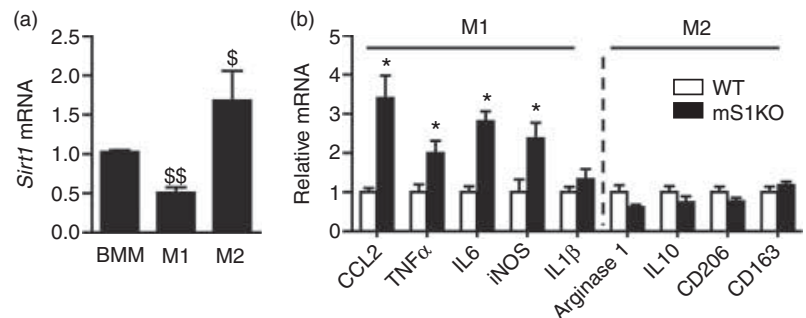

(c)

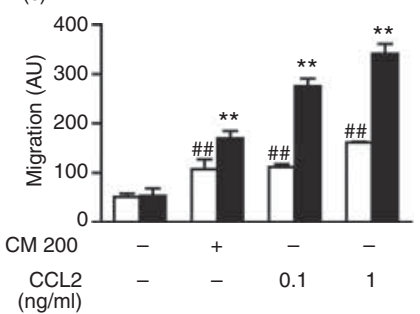

(d)

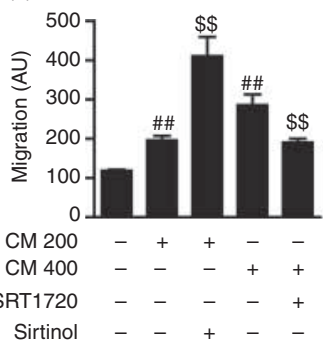

Figure 5

Regulation of macrophage polarization by SIRT1. (a) BMMs were treated with either $10 \mathrm{ng} / \mathrm{ml}$ LPS and $50 \mathrm{U} / \mathrm{ml} \mathrm{IFN} \gamma$ (for M1 polarization) or $10 \mathrm{ng} / \mathrm{ml}$ IL4 (for M2 polarization) for $24 \mathrm{~h}$. Sirt1 mRNA was measured by real-time RT-PCR. ${ }^{\$} P<0.05$ and ${ }^{\$ \$} P<0.01$ versus BMMs. (b) After M1 or M2 polarization, expression patterns of $\mathrm{M} 1$ and $\mathrm{M} 2$ markers were analyzed. (c) BMMs from WT or mS1KO mice were allowed to migrate through porous membranes for $3 \mathrm{~h}$ toward $200 \mu \mathrm{l}$ of adipocyte-conditioned medium (CM200) or CCL2. (d) RAW264.7 cells were treated with $10 \mathrm{nM}$ SRT 1720 or $2.5 \mu \mathrm{M}$ sirtinol for $24 \mathrm{~h}$ and migration toward adipocyte CM was determined. Cells in lower chambers were counted. Values are means \pm S.E.M. $(n=3)$. ${ }^{*} P<0.05$, and ${ }^{*} * P<0.01$ versus WT; ${ }^{\# \#} P<0.01$ versus vehicle; and ${ }^{\$} P<0.01$ versus $\mathrm{CM} 400$.

in activation of FAK, we conducted in vitro overexpression studies. Overexpression of SIRT1 in HEK293 cells decreased both the expression and acetylation of FAK, while overexpression of EP300 abolished these effects (Fig. 6c). In agreement with these findings, acetylation of FAK was increased in mS1KO cells, indicating that FAK is a SIRT1 substrate for deacetylation (Fig. 6d).

Finally, to confirm the role of FAK in adipocytemediated macrophage chemotaxis, we conducted a chemotaxis assay using RAW264.7 cells transfected with Ptk2 siRNA. Figure 6e shows that Ptk2 knockdown reduced migration of macrophages toward adipocyte CM. Moreover, sirtinol did not stimulate macrophage migration when Ptk2 was deleted, indicating that regulation of macrophage migration by SIRT1 might be mediated by FAK.

\section{Discussion}

As macrophage infiltration is a key component of obesity-induced tissue inflammation and SIRT1 has anti-inflammatory effects, we proposed the hypothesis that tissue inflammation and insulin resistance mediated by deletion of Sirt1 result from increased tissue infiltration by macrophages. Indeed, in several rodent models of obesity, deletion of myeloid Sirt1 promotes infiltration of macrophages into liver and adipose tissues, leading to impaired glucose homeostasis (Schug et al. 2010, Yang et al. 2012). Although these studies have dissected the mechanisms by which SIRT1 regulates tissue infiltration by macrophages and the subsequent insulin resistance, whether macrophage Sirt1 deletion affects the inflammation in the pancreas along with liver and adipose tissues, or how SIRT1 represses macrophage activation at the cellular level has not been studied. To address this question, we developed myeloid Sirt1-knockout mice and conducted in vitro experiments using BMMs from these knockout mice. We also investigated the effects of deletion of myeloid Sirt1 on pancreatic function, liver inflammation, and eWAT remodeling during HFD feeding.

ATMs are heterogeneous in their biological functions as well as the expression of cell-surface markers and are categorized into two subpopulations (Nguyen et al. 2007, Olefsky \& Glass 2010). The first are classically activated M1 macrophages that exert proinflammatory effects by expressing proinflammatory genes, such as Il1 $\beta$ (Il1b), Tnf, Il6, and Nos2, and infiltrate liver and adipose tissue in obesity. The other group of macrophages are alternatively activated or M2 macrophages that exert anti-inflammatory effects by expressing genes such as Il10, Arg1, and Mrc1 (Olefsky \& Glass 2010). Results from our in vitro experiments that expression of SIRT1 was lower in M1 macrophages and higher in M2 macrophages, and that deletion of myeloid Sirt1 stimulated M1 polarization. Consistently, mS1KO mice displayed increased ATM infiltration by $\mathrm{F} 4 / 80^{+} \mathrm{CD} 11 \mathrm{~b}^{+} \mathrm{CD} 11 \mathrm{c}^{+}$triply positive cells after HFD feeding. These results indicated that Sirt1 deficiency promoted a phenotypic switch of ATMs to a more proinflammatory M1 subtype. This finding is fully consistent with a previous report that treatment with a pharmacological SIRT1 activator reduced CD11c ${ }^{+}$M1 ATMs in obese rats (Yoshizaki et al. 2010). We also observed that genetic or pharmacological inhibition of SIRT1 in macrophages further stimulated cell migration in response to adipose chemoattractants; however, treatment of cells with SRT1720 hampered adipocyte CM-triggered macrophage migration.

The results of cellular signaling analysis provided evidence of the importance of SIRT1 for the modulation of the activation of NFKB1 and expression of FAK, and control of cell migration by FAK. Firstly, deletion of Sirt1 


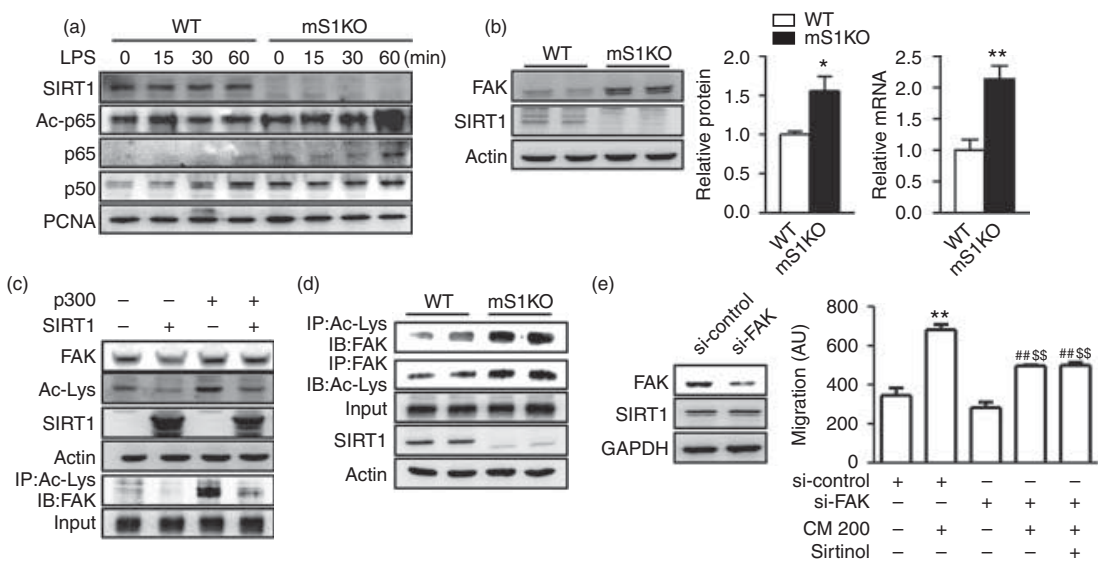

Figure 6

Regulation of NFKB1/FAK signaling pathways by SIRT1. (a) Immunoblots were performed on whole-cell extracts (for SIRT1) or nuclear extracts (for Ac-p65, p65, p50, and PCNA) from BMMs that had been treated with LPS for the indicated periods. (b) BMMs were isolated from WT or mS1KO mice, and protein and mRNA levels of FAK were measured. Values are means \pm S.E.M. $(n=3) .{ }^{*} P<0.05$ and ${ }^{*} * P<0.01$ versus WT. (c) HEK293 cells were transfected with p300 or SIRT1 and FAK expression and acetylation states

increased nuclear translocation and acetylation of NFKB1 subunits in mS1KO macrophages. Secondly, SIRT1 interacted with and deacetylated FAK, whereas acetyltransferase EP300 had the opposite effects. Thirdly, deletion of Sirt1 increased acetylation and expression of FAK in mS1KO macrophages. Fourthly, knockdown of Ptk2 in RAW264.7 cells impaired chemotaxis. These results indicate that, in addition to NFKB1, FAK might be a direct SIRT1 deacetylation substrate. In support of this possibility, results from previous studies have indicated that NFKB1 and FAK activate signaling for macrophage migration (Parsons 2003, Maa et al. 2008).

We expanded our understanding of the role of myeloid SIRT1 in vivo by demonstrating that $\mathrm{mS} 1 \mathrm{KO}$ mice exhibit tissue inflammation with increased infiltration of macrophages into the pancreas. We demonstrated that macrophage SIRT1 regulates pancreatic $\beta$-cell function and insulin secretion. As CCL2 is a potent macrophage chemoattractant, increased expression of CCL2 together with its receptor CCR2 could directly trigger the recruitment of macrophages to pancreatic tissue. Infiltrated macrophages could, in turn, secrete a variety of cytokines, including chemokines that further induce inflammationrelated gene expression and promote local inflammatory responses, resulting in apoptotic pancreatic $\beta$-cell death and impaired glucose tolerance. Notably, gene expression analysis revealed that levels of CD11c (a marker for M1 macrophages) and F4/80 (a pan-marker for macrophages) were analyzed. (d) BMMs isolated from WT or mS1KO mice were used for immunoprecipitation and immunoblotting. (e) RAW264.7 cells were transfected with scrambled or Ptk2 siRNA and treated with $2.5 \mu \mathrm{M}$ sirtinol or left untreated. Migration toward $200 \mu \mathrm{l}$ of adipocyte-conditioned medium (CM200) was determined. Values are mean \pm s.E.M. $(n=3)$. $* * P<0.01$ versus si-control; ${ }^{\# \#} P<0.01$ versus si-FAK without CM200; and ${ }^{\$ \$} P<0.01$ versus si-control with $\mathrm{CM} 200$.

were increased in islets from mS1KO mice, indicating infiltration by M1 macrophages. These results were consistent with our in vitro data indicating preferential differentiation of M1 macrophages associated with deletion of Sirt1. In addition, gene expression and serum levels of IL1 $\beta$ and TNF $\alpha$ were much higher in $\mathrm{mS1KO}$ mice than in WT mice, supporting a close link between increased macrophage infiltration and tissue inflammation upon feeding with a HFD.

We also found that deletion of myeloid Sirt1 promoted migration of macrophages to the liver and adipose tissue in response to a HFD. Similarly, we observed an increase in inflammatory cells in histological analysis of liver and adipose tissues from mS1KO mice. Obesity-induced inflammation in adipose tissues involves increased expression of proinflammatory mediators and infiltration of macrophages into adipose tissue, where they surround dead adipocytes to form typical CLSs (Cinti et al. 2005). Deletion of myeloid Sirt1 in HFD-fed mice resulted in increased proinflammatory cytokines in the liver and adipose tissue and an increase in the number of CLSs. Notably, mS1KO mice on a HFD showed a decrease in hepatic triglycerides and steatosis and smaller adipocyte size compared with WT mice. These findings are in contrast to those from the study by Schug et al. (2010), in which mS1KO mice exhibited greater weight gain and adipose tissue mass. These opposite results may result from differences in the diet composition; we fed mice with

Published by Bioscientifica Ltd 
60\% HFD, whereas Schug and colleagues used 45\% HFD. A higher calorie diet might recruit more macrophages into adipose tissue, which, in turn, would secrete more cytokines that are responsible for tissue destruction. In support thereof, marked apoptotic cell death and tissue fibrosis were observed in $\mathrm{mS} 1 \mathrm{KO}$ mice, indicating that adipocytes were replaced by fibrotic tissue, resulting in smaller adipose mass.

In summary, this study demonstrated that myeloid SIRT1 affects the migration response of macrophages by modulating the NFKB1/FAK pathways. These results were recapitulated in vivo by showing that deletion of myeloid Sirt1 promotes the migration of macrophages toward the pancreas, liver, and adipose tissue during feeding with a HFD (Supplementary Fig. 4, see section on supplementary data given at the end of this article). SIRT1 is involved in a nutrient-sensing pathway and its activation might have beneficial effects by affecting cellular functions including inflammation and apoptosis. Therefore, regulation of myeloid SIRT1 through pharmacological activation or diet control could be a useful anti-inflammatory therapeutic strategy for treating obesity-related metabolic disease.

\section{Supplementary data}

This is linked to the online version of the paper at http://dx.doi.org/10.1530/ JOE-14-0527.

\section{Declaration of interest}

The authors declare that there is no conflict of interest that could be perceived as prejudicing the impartiality of the research reported.

\section{Funding}

This work was supported by the Bio \& Medical Technology Development Program (grant number 2012M3A9B2027975) and the Medical Research Center Program (grant number 2008-0062279) through the National Research Foundation (NRF) funded by the Korean government (Ministry of Science, Information and Communication Technology and Future Planning (MSIP)).

\section{Author contribution statement}

S-O K and M-Y S performed the experiments and analyzed the data. E J B and $\mathrm{B}-\mathrm{H} \mathrm{P}$ conceived the study concept, designed the experiments, and wrote the manuscript. All authors read and approved the final manuscript.

\section{References}

Arkan MC, Hevener AL, Greten FR, Maeda S, Li ZW, Long JM, WynshawBoris A, Poli G, Olefsky J \& Karin M 2005 IKK- $\beta$ links inflammation to http://joe.endocrinology-journals.org DOI: $10.1530 / J O E-14-0527$
() 2015 Society for Endocrinology Printed in Great Britain obesity-induced insulin resistance. Nature Medicine 11 191-198. (doi:10.1038/nm1185)

Chalkiadaki A \& Guarente L 2012 Sirtuins mediate mammalian metabolic responses to nutrient availability. Nature Reviews. Endocrinology $\mathbf{8}$ 287-296. (doi:10.1038/nrendo.2011.225)

Cinti S, Mitchell G, Barbatelli G, Murano I, Ceresi E, Faloia E, Wang S, Fortier M, Greenberg AS \& Obin MS 2005 Adipocyte death defines macrophage localization and function in adipose tissue of obese mice and humans. Journal of Lipid Research 46 2347-2355. (doi:10.1194/ jlr.M500294-JLR200)

Clement K, Viguerie N, Poitou C, Carette C, Pelloux V, Curat CA, Sicard A, Rome S, Benis A, Zucker JD et al. 2004 Weight loss regulates inflammation-related genes in white adipose tissue of obese subjects. FASEB Journal 18 1657-1669. (doi:10.1096/fj.04-2204com)

Golubovskaya V, Kaur A \& Cance W 2004 Cloning and characterization of the promoter region of human focal adhesion kinase gene: nuclear factor $\kappa \mathrm{B}$ and p53 binding sites. Biochimica et Biophysica Acta 1678 111-125. (doi:10.1016/j.bbaexp.2004.03.002)

Hotamisligil GS 2006 Inflammation and metabolic disorders. Nature 444 860-867. (doi:10.1038/nature05485)

Ichioka M, Suganami T, Tsuda N, Shirakawa I, Hirata Y, Satoh-Asahara N, Shimoda Y, Tanaka M, Kim-Saijo M, Miyamoto Y et al. 2011 Increased expression of macrophage-inducible C-type lectin in adipose tissue of obese mice and humans. Diabetes 60 819-826. (doi:10.2337/ db10-0864)

Kanda H, Tateya S, Tamori Y, Kotani K, Hiasa K, Kitazawa R, Kitazawa S, Miyachi H, Maeda S, Egashira K et al. 2006 MCP-1 contributes to macrophage infiltration into adipose tissue, insulin resistance, and hepatic steatosis in obesity. Journal of Clinical Investigation 116 1494-1505. (doi:10.1172/JCI26498)

Kleiner DE, Brunt EM, Van Natta M, Behling C, Contos MJ, Cummings OW, Ferrell LD, Liu YC, Torbenson MS, Unalp-Arida A et al. 2005 Design and validation of a histological scoring system for nonalcoholic fatty liver disease. Hepatology 41 1313-1321. (doi:10.1002/hep.20701)

Le KA, Mahurkar S, Alderete TL, Hasson RE, Adam TC, Kim JS, Beale E, Xie C, Greenberg AS, Allayee $\mathrm{H}$ et al. 2011 Subcutaneous adipose tissue macrophage infiltration is associated with hepatic and visceral fat deposition, hyperinsulinemia, and stimulation of NF- $\kappa$ B stress pathway. Diabetes 60 2802-2809. (doi:10.2337/db10-1263)

Lee JH, Song MY, Song EK, Kim EK, Moon WS, Han MK, Park JW, Kwon KB $\&$ Park BH 2009 Overexpression of SIRT1 protects pancreatic $\beta$-cells against cytokine toxicity by suppressing the nuclear factor- $\kappa \mathrm{B}$ signaling pathway. Diabetes 58 344-351. (doi:10.2337/db07-1795)

Lumeng CN, Bodzin JL \& Saltiel AR 2007 Obesity induces a phenotypic switch in adipose tissue macrophage polarization. Journal of Clinical Investigation 117 175-184. (doi:10.1172/JCI29881)

Maa MC, Chang MY, Chen YJ, Lin CH, Yu CJ, Yang YL, Li J, Chen PR, Tang CH, Lei HY et al. 2008 Requirement of inducible nitric-oxide synthase in lipopolysaccharide-mediated Src induction and macrophage migration. Journal of Biological Chemistry 283 31408-31416. (doi:10.1074/jbc.M801158200)

Nguyen MT, Favelyukis S, Nguyen AK, Reichart D, Scott PA, Jenn A, LiuBryan R, Glass CK, Neels JG \& Olefsky JM 2007 A subpopulation of macrophages infiltrates hypertrophic adipose tissue and is activated by free fatty acids via Toll-like receptors 2 and 4 and JNK-dependent pathways. Journal of Biological Chemistry 282 35279-35292. (doi:10.1074/jbc.M706762200)

Olefsky JM \& Glass CK 2010 Macrophages, inflammation, and insulin resistance. Annual Review of Physiology 72 219-246. (doi:10.1146/ annurev-physiol-021909-135846)

Parsons JT 2003 Focal adhesion kinase: the first ten years. Journal of Cell Science 116 1409-1416. (doi:10.1242/jcs.00373)

Schug TT, Xu Q, Gao H, Peres-da-Silva A, Draper DW, Fessler MB, Purushotham A \& Li X 2010 Myeloid deletion of SIRT1 induces inflammatory signaling in response to environmental stress. Molecular and Cellular Biology 30 4712-4721. (doi:10.1128/MCB.00657-10) 
Shen Z, Ajmo JM, Rogers CQ, Liang X, Le L, Murr MM, Peng Y \& You M 2009 Role of SIRT1 in regulation of LPS- or two ethanol metabolitesinduced TNF- $\alpha$ production in cultured macrophage cell lines. American Journal of Physiology. Gastrointestinal and Liver Physiology 296 G1047-G1053. (doi:10.1152/ajpgi.00016.2009)

Solinas G, Vilcu C, Neels JG, Bandyopadhyay GK, Luo JL, Naugler W, Grivennikov S, Wynshaw-Boris A, Scadeng M, Olefsky JM et al. 2007 JNK1 in hematopoietically derived cells contributes to diet-induced inflammation and insulin resistance without affecting obesity. Cell Metabolism 6 386-397. (doi:10.1016/j.cmet.2007.09.011)

Suganami T, Tanimoto-Koyama K, Nishida J, Itoh M, Yuan X, Mizuarai S, Kotani H, Yamaoka S, Miyake K, Aoe S et al. 2007 Role of the Toll-like receptor $4 / \mathrm{NF}-\kappa \mathrm{B}$ pathway in saturated fatty acid-induced inflammatory changes in the interaction between adipocytes and macrophages. Arteriosclerosis, Thrombosis, and Vascular Biology $\mathbf{2 7}$ 84-91. (doi:10.1161/01.ATV.0000251608.09329.9a)
Weisberg SP, Hunter D, Huber R, Lemieux J, Slaymaker S, Vaddi K, Charo I, Leibel RL \& Ferrante AW Jr 2006 CCR2 modulates inflammatory and metabolic effects of high-fat feeding. Journal of Clinical Investigation $\mathbf{1 1 6}$ 115-124. (doi:10.1172/JCI24335)

Yang Z, Wang X, He Y, Qi L, Yu L, Xue B \& Shi H 2012 The full capacity of AICAR to reduce obesity-induced inflammation and insulin resistance requires myeloid SIRT1. PLOS ONE 7 e49935. (doi:10.1371/journal. pone.0049935)

Yoshizaki T, Schenk S, Imamura T, Babendure JL, Sonoda N, Bae EJ, Oh DY, Lu M, Milne JC, Westphal C et al. 2010 SIRT1 inhibits inflammatory pathways in macrophages and modulates insulin sensitivity. American Journal of Physiology 298 E419-E428. (doi:10.1152/ajpendo.00417.2009)

Zhang R, Chen HZ, Liu JJ, Jia YY, Zhang ZQ, Yang RF, Zhang Y, Xu J, Wei YS, Liu DP et al. 2010 SIRT1 suppresses activator protein-1 transcriptional activity and cyclooxygenase-2 expression in macrophages. Journal of Biological Chemistry 285 7097-7110. (doi:10.1074/jbc.M109.038604)

Received in final form 18 October 2014

Accepted 27 October 2014

Accepted Preprint published online 27 October 2014
(C) 2015 Society for Endocrinology Printed in Great Britain
Published by Bioscientifica Ltd. 